\title{
Density scaling and relaxation of the Pauli principle
}

\author{
Robert C. Morrison \\ Department of Chemistry, East Carolina University, Greenville, North Carolina 27858 \\ Paul W. Ayers \\ Department of Chemistry, McMaster University, Hamilton, Ontario L8S 4M1, Canada \\ Á Nagy \\ Department of Theoretical Physics, University of Debrecen, H-4010 Debrecen, Hungary and Atomic and \\ Molecular Physics Research Group of the Hungarian Academy of Sciences, H-4010 Debrecen, \\ Hungary
}

(Received 6 November 2006; accepted 30 January 2007; published online 30 March 2007)

\begin{abstract}
The relaxation of the Pauli principle associated with density scaling is examined. Scaling the density has been investigated in the development of density functional computational methods with higher accuracy. Scaling the density by $\rho(\mathbf{r})_{\zeta}=\rho(\mathbf{r}) / \zeta$ reduces the number of electrons to $M=N / \zeta$ when $\zeta>1$. The minimum kinetic energy of the scaled density, $T_{s}[\rho / \zeta]$, can be scaled back to the $N$-electron system by multiplying the $M$-electron Kohn-Sham-type occupation numbers by $\zeta$ to produce $T_{\zeta}[\rho]$. This relaxes the Pauli principle when the orbital occupation numbers are greater than 1 in the $N$-electron system. The effects of antisymmetry on solutions to the Kohn-Sham equations are examined for $\mathrm{Ne}$ and the Be isoelectronic series. The changes in $T_{\zeta}[\rho]$ and the exchange energy $E_{\mathrm{x} \zeta}[\rho]$ when $\zeta$ is varied show that these two quantities are inextricably linked. (C) 2007 American Institute of Physics. [DOI: 10.1063/1.2711190]
\end{abstract}

\section{INTRODUCTION}

A persistent challenge in approximate density functional theory (DFT) is the construction of density functionals that are free of self interaction. ${ }^{1-5}$ Hartree-Fock calculations are made self-interaction-free by the exchange energy which has terms that cancel the Coulomb self-interaction terms. Since the Hartree-Fock energy expression corresponds to the energy of a specific antisymmetric wave function, Hartree-Fock provides a self-interaction-free description. The challenge in DFT is to embed the antisymmetry information into approximate density functionals. Although this has been at least partially accomplished by approximate models ${ }^{4,6}$ including those that incorporate exchange, there is still work to be done.

Progress has been made in improving approximate functionals through the development of simple mathematical relationships that must be satisfied by functionals. ${ }^{1,2}$ Several such relationships are exemplified in the landmark paper on coordinate scaling by Levy and Perdew. ${ }^{7}$ Much recent work has focused on homogeneity properties of the density ${ }^{8-12}$ and on density scaling, ${ }^{13-15}$ i.e., $\rho_{\zeta}(\mathbf{r})=\rho(\mathbf{r}) / \zeta$. Such studies yield additional mathematical relationships for density functionals and may improve accuracy.

Nagy ${ }^{15}$ has used scaled densities for a series of atoms Be through Ar to calculate total energies within $2 \mathrm{~m}$ hartrees of the exact total energy for Be and within $115 \mathrm{~m}$ hartrees for Ar. The exchange energy $E_{x}$, the Kohn-Sham kinetic energy $T_{s}$, and the total energy are determined for an $M$-electron system, $M=N / \zeta$, using the density $\rho_{\zeta}(\mathbf{r})$, then scaling these quantities back to the $N$-electron system. Because the $M$-electron system consists of fully occupied $\left(n_{i}=1\right)$ or fully unoccupied $\left(n_{i}=0\right)$ spin-orbitals for a single determinant in the Kohn-Sham scheme or for members of an ensemble when there are fractional occupation numbers, the Pauli principle is violated in the $N(=M \zeta)$-electron system.

In this article we study the effect of relaxing the Pauli exclusion principle through the use of density scaling. Varying the scale factor $\zeta>1$ for a series of calculations changes the degree of relaxation of the Pauli principle. The quantities to be examined include the kinetic energy, the exchange energy, and the correlation energy. How are these quantities affected by the Pauli principle? We believe that the mathematical relationships derived from these studies may be especially helpful for studying self-interaction error which is, at its heart, due to the inability of many existing functionals to treat antisymmetry (i.e., the Fermi hole) successfully.

The energy as a functional of the density can be determined by a constrained search, ${ }^{16}$

$$
E[\rho]=\min _{\Psi \rightarrow \rho}\left\langle\Psi\left|\hat{T}+\hat{V}_{e e}\right| \Psi\right\rangle+\int \rho(\mathbf{r}) v(\mathbf{r}) d \mathbf{r},
$$

where $\hat{T}$ is the kinetic energy operator and $\hat{V}_{e e}$ is the electronelectron interaction. A constrained search over the kinetic energy results in the Kohn-Sham kinetic energy

$$
T_{s}[\rho]=\underset{\Psi \rightarrow \rho}{\min }\langle\Psi|\hat{T}| \Psi\rangle .
$$

While the minimum in this equation exists ${ }^{17}$ for densities that are noninteracting $v$-representable, sometimes a Slater determinant does not satisfy the Aufbau principle, ${ }^{18}$ resulting in holes below the Fermi level. The Aufbau principle can be retained if one relaxes the constraint that all of the spinorbitals be occupied $\left(n_{i}=1\right)$ or unoccupied $\left(n_{i}=0\right)$ and allows fractional occupation numbers. ${ }^{19,20}$ The minimization for 
systems that are not noninteracting $v$-representable can be achieved by generalizing the search to use density matrices corresponding to mixed states ${ }^{21-23}$

$$
\Gamma\left(\mathbf{x}_{1}^{\prime}, \ldots, \mathbf{x}_{N}^{\prime} \mid \mathbf{x}_{1}, \ldots, \mathbf{x}_{N}\right)=\sum_{i} w_{i} \Gamma_{i}\left(\mathbf{x}_{1}^{\prime}, \ldots, \mathbf{x}_{N}^{\prime} \mid \mathbf{x}_{1}, \ldots, \mathbf{x}_{N}\right),
$$

where

$$
\begin{aligned}
& \Gamma_{i}\left(\mathbf{x}_{1}^{\prime}, \ldots, \mathbf{x}_{N}^{\prime} \mid \mathbf{x}_{1}, \ldots, \mathbf{x}_{N}\right) \\
& \quad=\left|\Psi_{i}\left(\mathbf{x}_{1}, \ldots, \mathbf{x}_{N}\right)\right\rangle\left\langle\Psi_{i}\left(\mathbf{x}_{1}^{\prime}, \ldots, \mathbf{x}_{N}^{\prime}\right)\right|,
\end{aligned}
$$

and $0 \leqslant w_{i} \leqslant 1$, with $\sum_{i} w_{i}=1$. The notation $\mathbf{x}_{i}$ represents the space and spin coordinates for the ith electron, and $\mathbf{r}_{i}$ represents the spatial coordinates. For noninteger particle number this could be extended to grand canonical ensembles. ${ }^{1,22-25}$ The constrained search over ensembles takes the form

$$
E[\rho]=\min _{\Gamma^{\min } \rightarrow \rho} \operatorname{Tr}\left[\Gamma^{\min }\left(\hat{T}+\hat{V}_{e e}\right)\right]+\int \rho(\mathbf{r}) v(\mathbf{r}) d \mathbf{r},
$$

and

$$
T_{s}[\rho]=\min _{\Gamma^{0, \min } \rightarrow \rho} \operatorname{Tr}\left[\Gamma^{0, \min } \hat{T}\right] .
$$

The ensemble density matrix that minimizes $T_{s}[\rho]$ is $\Gamma^{0, \min }$. The total density can be expressed as

$$
\rho(\mathbf{r})=\sum_{i} w_{i} \rho_{i}(\mathbf{r})
$$

where

$$
\rho_{i}(\mathbf{r})=\operatorname{Tr}\left[\Gamma_{i} \hat{\rho}\right] .
$$

The total energy expression can be partitioned as

$$
E[\rho]=T_{s}[\rho]+\int \rho(\mathbf{r}) v(\mathbf{r}) d \mathbf{r}+J[\rho]+E_{\mathrm{xc}}[\rho],
$$

where $J[\rho]$ is the Coulomb energy and $E_{\mathrm{xc}}[\rho]$ is the exchange-correlation energy. The Kohn-Sham equations ${ }^{26}$ are

$$
\left[-\frac{1}{2} \nabla^{2}+v_{\mathrm{KS}}(\mathbf{r})\right] \phi_{i}=\varepsilon_{i} \phi_{i},
$$

where the Kohn-Sham potential is

$$
v_{\mathrm{KS}}(\mathbf{r})=v(\mathbf{r})+v_{J}(\mathbf{r})+v_{\mathrm{xc}}(\mathbf{r}) .
$$

The modified Kohn-Sham equations for the $M$-electron system are

$$
\left[-\frac{1}{2} \nabla^{2}+v_{\zeta \mathrm{KS}}(\mathbf{r})\right] \phi_{\zeta i}=\varepsilon_{\zeta i} \phi_{\zeta i},
$$

where the Kohn-Sham potential with the scaled reference density is

$$
v_{\zeta \mathrm{KS}}(\mathbf{r})=v_{\zeta}(\mathbf{r})+v_{J}(\mathbf{r})+v_{\zeta \mathrm{xc}}(\mathbf{r}) .
$$

The potentials $v_{\zeta}(\mathbf{r}), v_{J}(\mathbf{r}), v_{\zeta \mathrm{xc}}(\mathbf{r})$ are, respectively, the modified external potential, Coulomb potential, and modified exchange-correlation potential for the scaled density. The density $\rho_{\zeta}(\mathbf{r})$ is given by

$$
\rho_{\zeta}(\mathbf{r})=\sum_{i=1}^{m} n_{\zeta i}\left|\varphi_{\zeta i}(\mathbf{r})\right|^{2},
$$

and the kinetic energy $T_{s}[\rho / \zeta]$ is given by

$$
T_{s}[\rho / \zeta]=\sum_{i=1}^{m}-\frac{1}{2} n_{\zeta i}\left\langle\phi_{\zeta i}\left|\nabla^{2}\right| \phi_{\zeta i}\right\rangle .
$$

The summation in Eqs. (14) and (15) is over the $m$ spatial orbitals that have nonzero occupation. The $n_{\zeta i}$ are the spintraced occupation numbers for the $M$-electron system, $n_{\zeta i}$ $\leqslant 2$. For the $N$-electron singlet state when $M=2, \phi_{\zeta i}$ $=(\rho(\mathbf{r}) / N)^{1 / 2}, m=1$ and $n_{\zeta i}=2$. The resulting kinetic energy is then scaled back to the $N$-electron system by multiplying the occupation numbers $n_{\zeta i}$ by $\zeta$,

$$
T_{\zeta}[\rho]=\zeta T_{s}[\rho / \zeta] \text {. }
$$

The $N$-electron density in terms of the $M$-electron occupation numbers and orbitals is

$$
\rho(\mathbf{r})=\sum_{i=1}^{m} \zeta n_{\zeta i}\left|\phi_{\zeta i}(\mathbf{r})\right|^{2}=\sum_{i=1}^{m} n_{i}\left|\phi_{\zeta i}(\mathbf{r})\right|^{2},
$$

where $n_{i}=\zeta n_{\zeta i}$ are the spin-traced occupation numbers scaled back to the $N$-electron system.

The Kohn-Sham equations are solved for a reference density $\rho(\mathbf{r})$ using the Zhao-Morrison-Parr (ZMP) method. ${ }^{27-29}$ Accurate densities and total energies are obtained from accurate configuration interaction (CI) calculations. The accurate $N$-electronic densities are scaled by $\zeta>1$, to give $M=N / \zeta$ electrons. The modified Kohn-Sham equations, Eq. (12), are solved using the scaled $M$-electron density as the reference density. The occupation numbers $n_{\zeta i}$ are varied to minimize $T_{s}[\rho / \zeta]$ for the $M$-electron system with the occupation numbers restricted to satisfy the Pauli exclusion principle for the M-electron system, $n_{\zeta i} \leqslant 2$. The obtained fractional occupation numbers represent some ensemble density that correspond to antisymmetric wave functions, as in Eqs. (3) and (4). When these occupation numbers are scaled back to the $N$-electron system the resulting ensemble density does not generally correspond to an ensemble density for which all terms correspond to antisymmetric wave functions. So the Pauli principle is relaxed for the resulting $N$-electron system. The total energy of the CI wave function is used in calculating the correlation and exchangecorrelation energies.

The scaled density $\rho_{\zeta}(\mathbf{r})$ has the same shape function $\sigma(\mathbf{r})$ as $\rho(\mathbf{r})$ but with fewer electrons $M=N / \zeta$ when $\zeta<1$. The shape function is defined as ${ }^{30,31}$

$$
\rho(\mathbf{r})=N \sigma(\mathbf{r}) .
$$

Although the shape function determines the external potential and number of electrons for Coulombic systems, ${ }^{31}$ this mapping cannot be used because the external potential $v_{\zeta}(\mathbf{r})$ is not Coulombic except when $\zeta=1$.

\section{DENSITY SCALING AND ANTISYMMETRY}

The $T_{s}[\rho / \zeta]$ obtained from the constrained search for the reference density $\rho(\mathbf{r}) / \zeta$ can be scaled back to the $N$-electron 


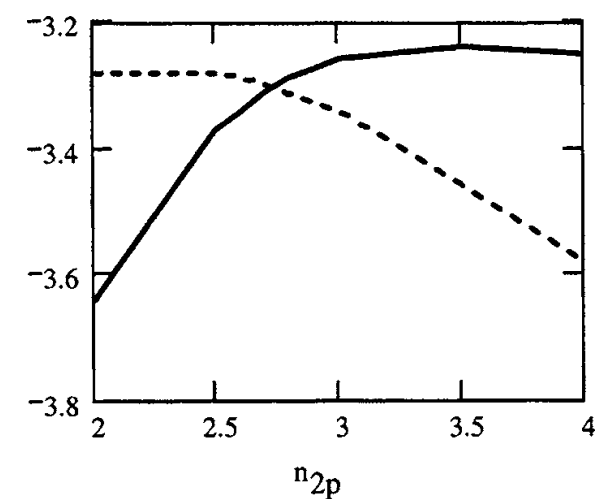

FIG. 1. Plots of the eigenvalues for the $2 p$ (solid line) and $2 s$ (dashed line) orbitals for neon with $M=6$.

system ${ }^{13}$ using Eq. (16). This is equivalent to multiplying the occupation numbers in Eqs. (14) and (15) by $\zeta$, producing the original density $\rho(\mathbf{r})$ in Eq. (17). This gives spin-traced occupations greater than two and hence violates the Pauli exclusion principle for the corresponding Kohn-Sham $\mathrm{N}$-electron wave function or ensemble.

Here we distinguish between two "Kohn-Sham" systems: the one having the scaled density (this is the "generalized Kohn-Sham scheme" of Refs. 14 and 15) and the one having the original density, that is, the density scaled back to the original density. In the former scheme the Pauli principle is valid. In the latter, which we study in this paper, the Pauli principle is (partially) relaxed.

The Chan-Handy ${ }^{13}$ examples for neon use $\zeta=N / M$ with $N=10$ and $M=2,4,6$, or 8 . We have computed the occupation numbers and $T_{s}[\rho / \zeta]$ for the $M$-electron systems using the ZMP method ${ }^{27-29}$ using a previously computed electron density $^{32}$ for neon. The occupation numbers were optimized by minimizing $T_{s}[\rho / \zeta]$. The occupation numbers for these $M$-electron systems were not allowed to violate the Pauli exclusion principle; double occupancy is not exceeded for any spatial orbital. (The maximum allowed occupancies were $n_{\zeta 1 s}=n_{\zeta 2 s}=2$ and $n_{\zeta 2 p}=6$ for the scaled densities $\rho(\mathbf{r}) / \zeta)$.

An example of the optimization of $T_{s}[\rho / \zeta]$ is illustrated for $M=6$ and $N=10$. The $2 s$ and $2 p$ eigenvalues are inverted from their normal relative values for $n_{2 p}<2.73$. The minimum $T_{s}$ occurs at the point where the $2 s$ and $2 p$ eigenvalue curves cross at $n_{2 p}=2.73$. Graphs of orbital eigenvalues $\varepsilon_{2 s}$ and $\varepsilon_{2 p}$ versus the occupation number $n_{2 p}$ are given in Fig. 1. The graph of $T_{s}$ versus the $2 p$ occupation number for $M=6$ is given in Fig. 2.

The occupation numbers scaled back to the 10-electron neon atom are $n_{1 s}=10$ for $M=2 ; n_{1 s}=5$ and $n_{2 p}=5$ for $M$ $=4 ; n_{1 s}=3.333, n_{2 s}=2.1167$, and $n_{2 p}=4.55$ for $M=6$; and $n_{1 s}=2.5, n_{2 s}=2.5$, and $n_{2 p}=5.0$ for $M=8$. When $M=4, \varepsilon_{2 p}$ $<\varepsilon_{2 s}$ for all occupation numbers is allowed by the Pauli principle. The above occupation numbers result in the same spatial orbital being occupied by more than two electrons, at least partially reducing antisymmetry in the resulting ensemble.

Though generally not unique, the following lists some examples of $N$-electron ensembles that would produce the above occupations:

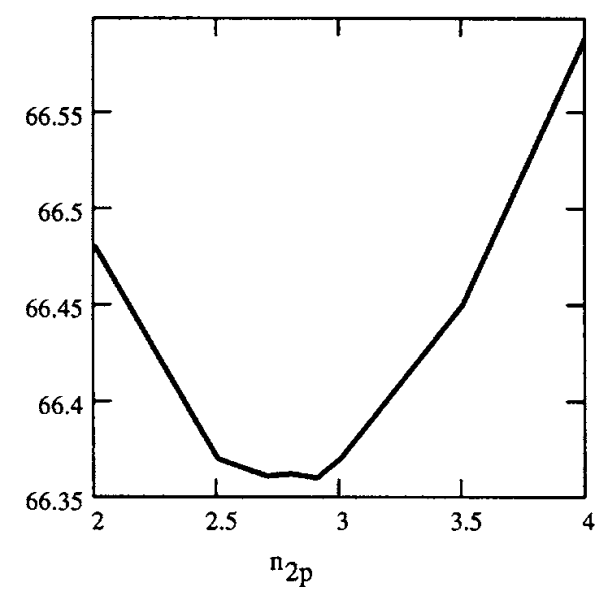

FIG. 2. Plot of $T_{s}$ vs $n_{2 p}$ for the neon atom with $M=6$.

$$
\begin{aligned}
\Gamma(M=2)= & \left|\Psi\left(1 s^{10}\right)\right\rangle\left\langle\Psi\left(1 s^{10}\right)\right|, \\
\Gamma(M=4)= & \frac{1}{2}\left|\Psi\left(1 s^{4} 2 p^{6}\right)\right\rangle\left\langle\Psi\left(1 s^{4} 2 p^{6}\right)\right| \\
& +\frac{1}{2}\left|\Psi\left(1 s^{6} 2 p^{4}\right)\right\rangle\left\langle\Psi\left(1 s^{6} 2 p^{4}\right)\right|, \\
\Gamma(M=6)= & \frac{1}{3}\left|\Psi\left(1 s^{2} 2 s^{2} 2 p^{6}\right)\right\rangle\left\langle\Psi\left(1 s^{2} 2 s^{2} 2 p^{6}\right)\right| \\
& +0.55\left|\Psi\left(1 s^{4} 2 s^{2} 2 p^{4}\right)\right\rangle\left\langle\Psi\left(1 s^{4} 2 s^{2} 2 p^{4}\right)\right| \\
& +0.1167\left|\Psi\left(1 s^{4} 2 s^{3} 2 p^{3}\right)\right\rangle\left\langle\Psi\left(1 s^{4} 2 s^{3} 2 p^{3}\right)\right|,
\end{aligned}
$$

$$
\begin{aligned}
\Gamma(M=8)= & \frac{1}{2}\left|\Psi\left(1 s^{2} 2 s^{2} 2 p^{6}\right)\right\rangle\left\langle\Psi\left(1 s^{2} 2 s^{2} 2 p^{6}\right)\right| \\
& +\frac{1}{2}\left|\Psi\left(1 s^{3} 2 s^{3} 2 p^{4}\right)\right\rangle\left\langle\Psi\left(1 s^{3} 2 s^{3} 2 p^{4}\right)\right| .
\end{aligned}
$$

The $M$ value for each of the $N$-electron $\Gamma$ s indicates the scaling used to solve the $M$-electron Kohn-Sham equations for the density $\rho(\mathbf{r}) / \zeta$. The orbitals used for the $N$-electron system are those determined for the $M$-electron system by using the scaled density as the reference density. In each case the obtained density is $\rho(\mathbf{r})$. The first term for $\Gamma(M=6)$ and $\Gamma(M=8)$ is antisymmetric, but the subsequent terms are not antisymmetric. Neither of the terms in $\Gamma(M=4)$ is antisymmetric, and in $\Gamma(M=2)$ the same spatial orbital is occupied by all ten of the electrons.

\section{RELAXING ANTISYMMETRY IN THE BERYLLIUM ISOELECTRONIC SERIES}

The beryllium isoelectronic series ground states provide a relatively simple expression for the ensemble density that results from scaling the density. The antisymmetric configurations in the ensemble that produces $T_{s}[\rho / \zeta]$ for the scaled density $\rho(\mathbf{r}) / \zeta$ correspond to occupation numbers $n_{\zeta 1 s}=2$ and $n_{\zeta 2 s}=2(2-\zeta)$, as determined by obtaining the minimum kinetic energy $T_{s}[\rho / \zeta]$. The occupation numbers of the Kohn- 
Sham orbitals that produce this $T_{s}[\rho / \zeta]$ are then scaled back to the $N$-electron system giving $n_{1 s}=2 \zeta$ and $n_{2 s}=2(2-\zeta) \zeta$. For example, when $\zeta=4 / 3$ the computed $T_{s}[\rho / \zeta]$ is for the three-electron system that has the density $3 \rho(\mathbf{r}) / 4$. The resulting occupation numbers scaled back to the four-electron system are $n_{1 s}=8 / 3$ and $n_{2 s}=4 / 3$.

A four-electron ensemble density matrix that gives the correct occupation numbers for a given scale factor $\zeta$ is

$$
\begin{aligned}
\Gamma_{\zeta}= & (2-\zeta)\left|\Psi\left(1 s^{2} 2 s^{2}\right)\right\rangle\left\langle\Psi\left(1 s^{2} 2 s^{2}\right)\right| \\
& +(\zeta-1)\left|\Psi\left(1 s^{4}\right)\right\rangle\left\langle\Psi\left(1 s^{4}\right)\right| .
\end{aligned}
$$

The wave function $\left|\Psi\left(1 s^{4}\right)\right\rangle$ is expressed as

$$
\begin{aligned}
\Psi\left(x_{1}, x_{2}, x_{3}, x_{4}\right) \\
=\left(\frac{1}{\sqrt{4 !} \sqrt{2} \sqrt{2}}\right) \sum_{p} \hat{P}\left(1 s\left(r_{1}\right) 1 s\left(r_{2}\right) 1 s\left(r_{3}\right) 1 s\left(r_{4}\right)\right. \\
\quad \times[\alpha(1) \alpha(2) \beta(3) \beta(4)] .
\end{aligned}
$$

To ensure indistinguishability of the electrons the sum is over the 4 ! permutations $\hat{P}$. All terms in this summation have a positive sign, so antisymmetry is excluded from this term in the ensemble. The $\sqrt{2}$ factors in the denominator account for over counting the spin products.

The contribution to the two-electron energy for this symmetric wave function is

$$
V_{12}=(1-1 / 4) J[\rho],
$$

where $-J / 4$ represents the Fermi-Amaldi correction for the two-electron energy when $N=4 .^{33,34}$ This leads us to express a weighted exchange energy, $E_{\mathrm{x} \zeta}^{(2)}$, for the ensemble,

$$
E_{\mathrm{x} \zeta}^{(2)}=w_{1} E_{\mathrm{x} \zeta}\left(1 s^{2} 2 s^{2}\right)+w_{2}(-J / 4),
$$

where $E_{\mathrm{x} \zeta}\left(1 s^{2} 2 s^{2}\right)$ is the exchange energy of the $1 s^{2} 2 s^{2}$ determinant for the scale factor $\zeta$. We will also study the results when only the first term of Eq. (26) is included and the exchange from the "boson" second term is ignored,

$$
E_{\mathrm{x} \zeta}^{(1)}=w_{1} E_{\mathrm{x} \zeta}\left(1 s^{2} 2 s^{2}\right),
$$

and the unweighted single determinantal exchange energy

$$
E_{\mathrm{x} \zeta}^{(0)}=E_{\mathrm{x} \zeta}\left(1 s^{2} 2 s^{2}\right) \text {. }
$$

The Hartree-Fock expression for the exchange energy is

$$
E_{x}[\gamma]=-\frac{1}{2} \int \frac{\left|\gamma\left(\mathbf{r}_{1}, \mathbf{r}_{2}\right)\right|^{2}}{\left|\mathbf{r}_{1}-\mathbf{r}_{2}\right|} d \mathbf{r}_{1} d \mathbf{r}_{2} .
$$

The corresponding expression for the pair density from the two matrix is

$$
\Gamma\left(x_{1} x_{2} \mid x_{1} x_{2}\right)=\frac{1}{2}\left[\gamma\left(x_{1} x_{1}\right) \gamma\left(x_{2} x_{2}\right)-\gamma\left(x_{1} x_{2}\right) \gamma\left(x_{2} x_{1}\right)\right] .
$$

When the occupation numbers of the spin-orbitals are different from 0 or 1 the resulting $\Gamma$ is not $N$ representable and has a trace greater than $N(N-1) / 2$ (but less than $N^{2} / 2$ ).

The $\mathrm{Be}, \mathrm{Ne}^{+6}, \mathrm{Ar}^{+14}, \mathrm{Kr}^{+32}$, and $\mathrm{Xe}^{+50}$ densities from previous work ${ }^{35}$ were used to compute $T_{s \zeta}, E_{\mathrm{x} \zeta}$, and $E_{c \zeta}$. Chan and Handy ${ }^{13}$ have shown

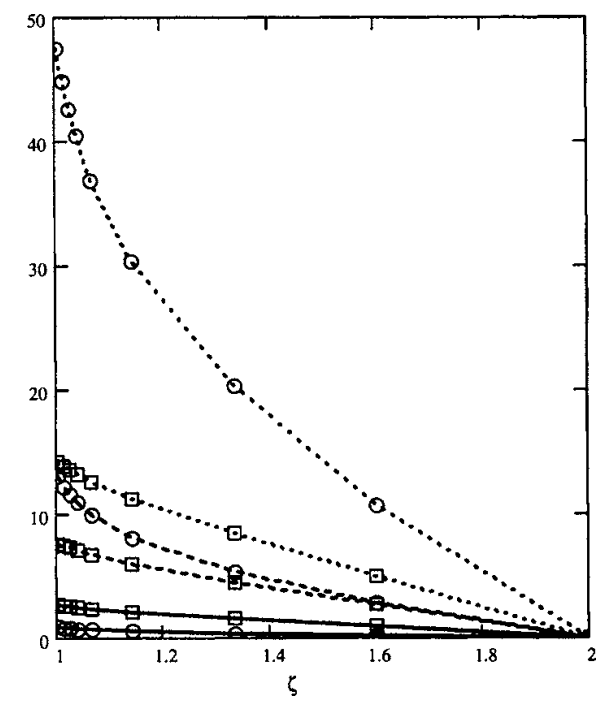

FIG. 3. $T_{\theta \zeta}$ for $\mathrm{Ar}^{+14}$ is the dotted line with circles. The dotted line with boxes is $\left|E_{\mathrm{x} \zeta}^{(1)}\right|$ for $\mathrm{Ar}^{+14}$. The dashed line with circles is $T_{\theta \zeta}$ for $\mathrm{Ne}^{+6}$, and the dashed line with boxes is $\left|E_{\mathrm{x} \zeta}^{(1)}\right|$ for $\mathrm{Ne}^{+6}$. The solid line with circles is $T_{\theta \zeta}$ for $\mathrm{Be}$, and the solid line with boxes is $\left|E_{\mathrm{x} \zeta}^{(1)}\right|$ for Be.

$$
T_{\zeta}[\rho] \leqslant T_{\zeta^{\prime}}[\rho]\left(\zeta \geqslant \zeta^{\prime} ; \zeta \geqslant 1\right) .
$$

This has also been illustrated in the work of Nagy. ${ }^{15}$ The equation indicates that the relaxing of the antisymmetry constraint on the density-matrix constrained search [Eq. (6)] lowers the kinetic energy $T_{\zeta}[\rho]$. When $\zeta=2$ the value of $T_{s \zeta}$ is the Weizsäcker kinetic energy $T_{W}$, which is known to be a lower bound to the true kinetic energy. ${ }^{36}$ It is also known that the essential error in the Weizsäcker-type functionals is due to the Pauli principle so that a correction termed the Pauli kinetic energy ${ }^{37-39}$ is required,

$$
T_{\theta}=T_{s}-T_{W}=T_{\zeta=1}[\rho]-T_{\zeta=2}[\rho] .
$$

It has been known for a long time $\mathrm{e}^{38,40,41}$ that the Pauli principle can simply be handled by a local potential. If there were no Pauli principle the kinetic energy would be the Weizsacker term. The effect of the antisymmetry of the wave function appears as a local potential ${ }^{42}$ in the Schrodinger type equation for the square root of the density.

This complete relaxation of the Pauli principle corresponds to the case $M=2$ (or $\zeta=N / 2$ ), when we have a single Kohn-Sham equation, the kinetic energy is the Weizsacker energy and the potential in this equation incorporates the Pauli potential. The present study makes it possible to relax the Pauli principle continuously. All cases when $\zeta<N / 2$ (but of course, $\zeta>1$ ) correspond to a partial relaxation of the Pauli principle. The density is determined by Eq. (17) with occupation numbers scaled back, that is, not satisfying the Pauli principle. The modified kinetic energy and consequently the modified Pauli energy [Eq. (32)] get smaller and the modified Kohn-Sham potential has a contribution from the partial relaxation of the Pauli principle.

The Pauli kinetic energy for the $T_{\zeta}[\rho]$ can be taken as

$$
T_{\theta \zeta}[\rho]=T_{\zeta}[\rho]-T_{W}[\rho] \text {. }
$$

In Fig. 3 the plots of $T_{\theta \zeta}$ and $\left|E_{\mathrm{x \zeta}}^{(1)}\right|$ for $\mathrm{Be}, \mathrm{Ne}^{+6}$, and $\mathrm{Ar}^{+14}$ are shown, where $E_{\mathrm{x} \zeta}^{(1)}=w_{1} E_{\mathrm{x} \zeta}\left(1 s^{2} 2 s^{2}\right)$ is the weighted ex- 
TABLE I. Kinetic energies for $\mathrm{Be}, \mathrm{Ne}^{+6}$, and $\mathrm{Ar}^{+14}$ for the BHHLYP and SLYP functionals, and the Weizsacker kinetic energy.

\begin{tabular}{llllll}
\hline \hline & $\mathrm{Be}$ & $\mathrm{Ne}^{+6}$ & $\mathrm{Ar}^{+14}$ & $\mathrm{Kr}^{+32}$ & $\mathrm{Xe}^{+50}$ \\
\hline$T_{s}$ & 14.59 & 110.16 & 377.5 & 1564.6 & 3561.5 \\
$T_{s}(\mathrm{BHHLYP})$ & 14.60 & 108.64 & 376.75 & 1515.5 & 3481.5 \\
$T_{s}(\mathrm{SLYP})$ & 14.42 & 107.98 & 375.12 & 1515.2 & 3478.5 \\
$T_{W}$ & 13.64 & 97.13 & 330.04 & 1344.4 & 3050.7 \\
$T_{s}(\mathrm{LLP})$ & 14.59 & 108.73 & 371.82 & 1532.7 & 3485.7 \\
\hline \hline
\end{tabular}

change energy from the single determinant. The Pauli kinetic energy and the magnitude of the exchange energy increase together, going from right to left in Fig. 3. Perdew et al. ${ }^{43}$ point out that a higher kinetic energy allows orbitals to dig a more local and deeper exchange hole. Here we see the kinetic energy increasing as $\left|E_{\mathrm{x} \zeta}^{(1)}\right|$ increases. We note the dramatic increase in the Pauli kinetic energy as the charge on the ions increases for the higher charged ions. The higher kinetic energy is necessary to dig the exchange hole for the more highly charged ions.

The importance of approximations to the exchange can also be illustrated by comparing the kinetic energies obtained from approximate functionals. In Table I we compare $T_{s}$ values obtained using the Slater exchange ${ }^{44}$ with the LYP (Ref. 45) correlation functional (SLYP) and the Becke $50 \%$ Hartree-Fock exchange with the Becke- 88 exchange ${ }^{46-48}$ and the LYP correlation functional (BHHLYP). The LYP functional is based on the Colle-Salvetti ${ }^{49,50}$ functional which is self-interaction-free by construction. These calculations using these approximate functionals were performed using GAMESS-USA (Ref. 51) with the standard 6-311G basis set for $\mathrm{Be}, \mathrm{Ne}^{+6}$, and $\mathrm{Ar}^{+14}$, and the MIDI basis set for $\mathrm{Kr}^{+32}$ and $\mathrm{Xe}^{+50}$. The Weizsacker kinetic energy, $T_{W}$ is included for comparison. Qualitatively one can observe from the results in Table I that a more accurate account of the antisymmetry of the wave function through the exchange functional produces a higher kinetic energy even for the approximate functionals, and a lower total energy is produced if the virial theorem holds for the calculation.

The conjointness ${ }^{52-55}$ of the kinetic and exchange energies shows the inextricable link between the kinetic and exchange energies. This is seen even in this setting where antisymmetry is being relaxed for these four-electron systems. Because of the connection to the conjointness we compare kinetic energies and exchange energies computed by the conjoined Becke and the Lee-Lee-Parr (LLP) equations for exchange and kinetic energies. Lee et al. ${ }^{52}$ use the Becke equation $^{46,47}$ for the exchange energy,

$$
E_{\mathrm{x}}(\text { Becke })=2^{1 / 3} C_{x} \int \sum_{\sigma} \rho_{\sigma}(\mathbf{r})\left[1+\beta G\left(x_{\sigma}\right)\right] d \mathbf{r},
$$

to determine an equation for the kinetic energy

$$
T_{s}(\mathrm{LLP})=2^{2 / 3} C_{F} \int \sum_{\sigma} \rho_{\sigma}(\mathbf{r})\left[1+\alpha G\left(x_{\sigma}\right)\right] d \mathbf{r} .
$$

The summation is over the spin $\sigma$. The constants are $C_{x}$ $=3 / 4(3 / \pi)^{1 / 3}, C_{F}=3 / 10\left(3 \pi^{2}\right)^{2 / 3}, \alpha=4.4188 \times 10^{-3}$, and $\beta$ $=4.5135 \times 10^{-3}$.

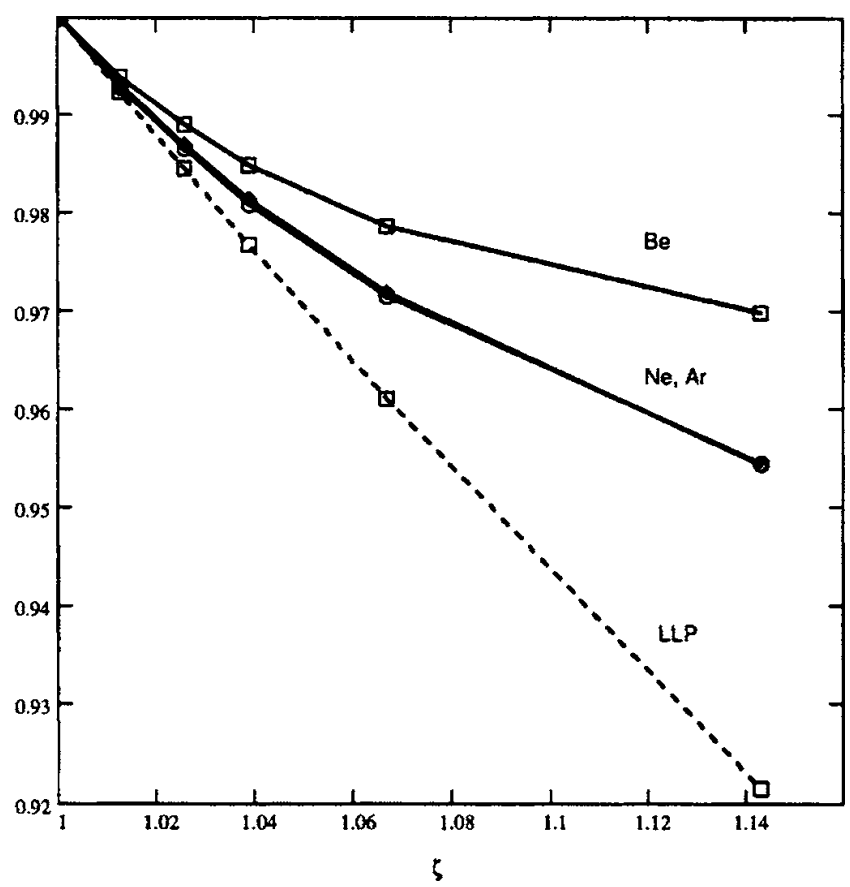

FIG. 4. The solid line with boxes is $T_{s \zeta} / T_{s \zeta=1}$ for Be. The solid line with circles (and superimposed diamonds) are the superimposed $T_{s \zeta} / T_{s \zeta=1}$ for $\mathrm{Ne}^{+6}$ and $\mathrm{Ar}^{+14}$. The dashed line with boxes are the $T_{s \zeta}(\mathrm{LLP}) / T_{s \zeta=1}(\mathrm{LLP})$ for $\mathrm{Be}, \mathrm{Ne}^{+6}$, and $\mathrm{Ar}^{+14}$.

The ratio of the kinetic energies $T_{s \zeta}[\rho] / T_{s \zeta=1}[\rho]$ are plotted in Fig. 4. The kinetic energies $T_{s}$ decrease, as expected, with increasing $\zeta$. The line representing $T_{s \zeta}($ LLP) deviates only slightly from $\zeta^{-2 / 3}$, because of the $\rho^{5 / 3}$ dependence in the integrand for $T_{s}$ (LLP). We use the scale factor of $\zeta$ to scale $T_{s \zeta}[\rho / \zeta]$ back to the $N$-electron system.

The values of the scale factor $\zeta_{0}$, which make $E_{\mathrm{c}}=0$ when computing the exchange from Eq. (29), become smaller as the nuclear charge increases for these fourelectron systems even though the correlation energy increases with nuclear charge with $\zeta=1$, as illustrated in Table II. The exchange energy and Becke exchange energy are also listed for $\zeta=1$ in Table II.

As $\zeta$ increases, the occupation of the $1 s$ orbital relative to the $2 s$ orbital increases and more of the burden of the density is shifted to the $1 s$ orbital. This decreases the Coulomb repulsion energy $J_{\zeta}(1 s)$ of electrons in the $1 s$ orbital, since the $1 s$ orbital must expand into the region normally associated with the $2 s$ orbital. The magnitude of the corresponding exchange energy of the $1 s^{2} 2 s^{2}$ determinant, $E_{\mathrm{x} \zeta}^{(0)}$ $=E_{\mathrm{x} \zeta}\left(1 s^{2} 2 s^{2}\right)$ is likewise reduced. These quantities are illustrated for the Be atom in Fig. 5. $J_{\zeta}(1 s)$ decreases while $J_{\zeta}(2 s)$

TABLE II. Values of $\zeta$ for which $E_{\mathrm{c}}=0$ and the exchange and correlation energies for $\zeta=1$.

\begin{tabular}{llllll}
\hline \hline & $\mathrm{Be}$ & $\mathrm{Ne}^{+6}$ & $\mathrm{Ar}^{+14}$ & $\mathrm{Kr}^{+32}$ & $\mathrm{Xe}^{+50}$ \\
\hline$\zeta_{0}$ & 1.0120 & 1.0028 & 1.0010 & 1.00044 & 1.00037 \\
$E_{\mathrm{c}}(\zeta=1)$ & -0.092 & -0.194 & -0.256 & -0.733 & -1.584 \\
$E_{x}$ & -2.67 & -7.60 & -14.15 & -28.91 & -43.65 \\
$E_{x}($ Becke $)$ & -2.67 & -7.59 & -14.13 & -28.88 & -43.63 \\
\hline \hline
\end{tabular}




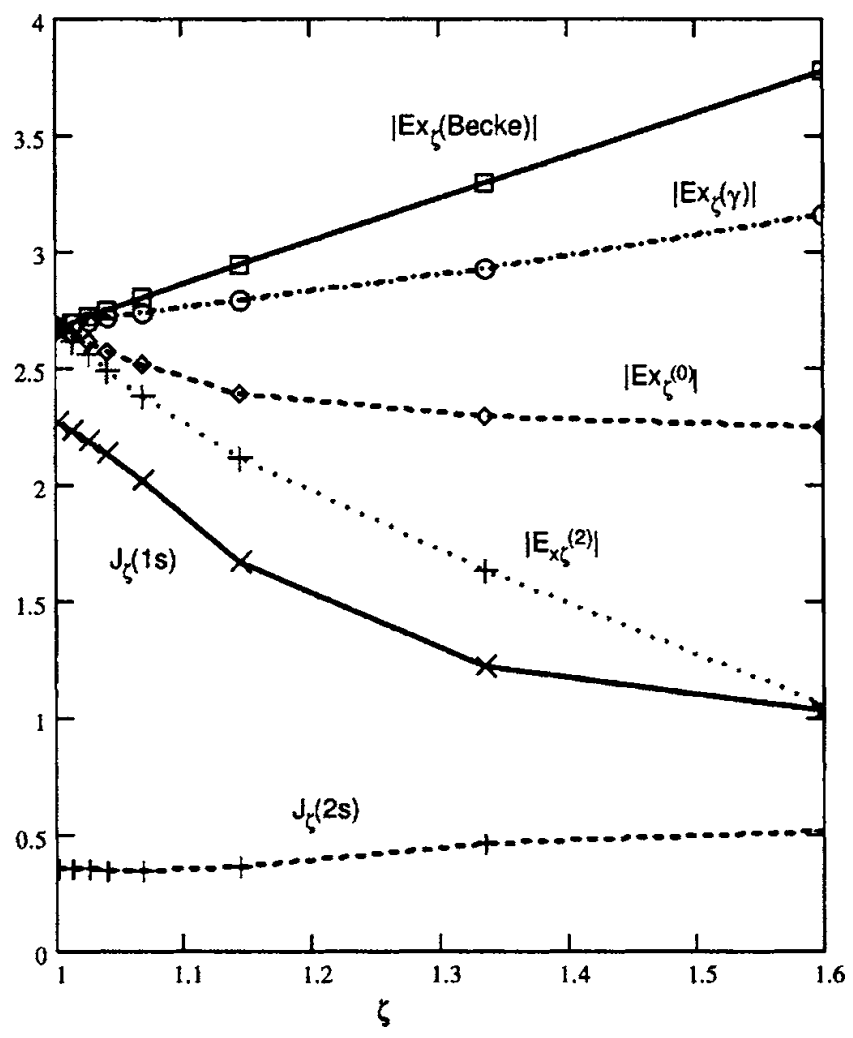

FIG. 5. Energy components for the Be atom. The dot-dashed line with circles is $\left|E_{\mathrm{x} \zeta}\right|$ from the 1-matrix. The dashed line with diamonds is $\left|E_{\mathrm{x} \zeta}^{(0)}\right|$ from the $1 s^{2} 2 s^{2}$ determinant. The solid line with x's is the Coulomb energy for the $1 s$ orbital. The dashed line with +'s is the Coulomb energy for the $2 s$ orbital. The dotted line with + 's is the magnitude of the weighted exchange $\left|E_{\mathrm{x} \zeta}^{(2)}\right|$. The solid line with boxes is $\left|E_{\mathrm{x} \zeta}\right|$ computed from the Becke formula.

increases slightly with $\zeta$. The magnitude of $E_{\mathrm{x} \zeta[}\left[1 s^{2} 2 s^{2}\right]$ also decreases. But, because the occupation numbers of the Kohn-Sham-type $1 s$ orbital increase and are now more than two, the magnitude of the exchange energy obtained from the 1-matrix increases.

Plots of the correlation energy obtained using various ways of computing the exchange for Be are compared in Fig. 6. The correlation energy is taken to be

$$
E_{\mathrm{c} \zeta}^{(n)}=E_{\mathrm{xc} \zeta}-E_{\mathrm{x} \zeta}^{(n)},
$$

where

$$
E_{\mathrm{xc} \zeta}=E-T_{s \zeta}-J-\int v(\mathbf{r}) \rho(\mathbf{r}) d \mathbf{r} .
$$

The superscript $(n)$ refers to the various ways of computing the exchange energy. The correlation energy using the Nagy formula for the exchange energy increases and crosses zero, forming the basis for using the density scaling to accurately determine the energy properties. The LYP correlation energy, ${ }^{45}$ included here for comparison, decreases nearly linearly toward zero. The correlation energy $E_{c \zeta}^{(1)}$ initially increases, but then decreases substantially as it accounts for an increasing amount of the non-Coulombic contributions brought in by the density scaling. It is noteworthy that the correlation determined using the Becke formula for exchange increases even more significantly than the correlation using

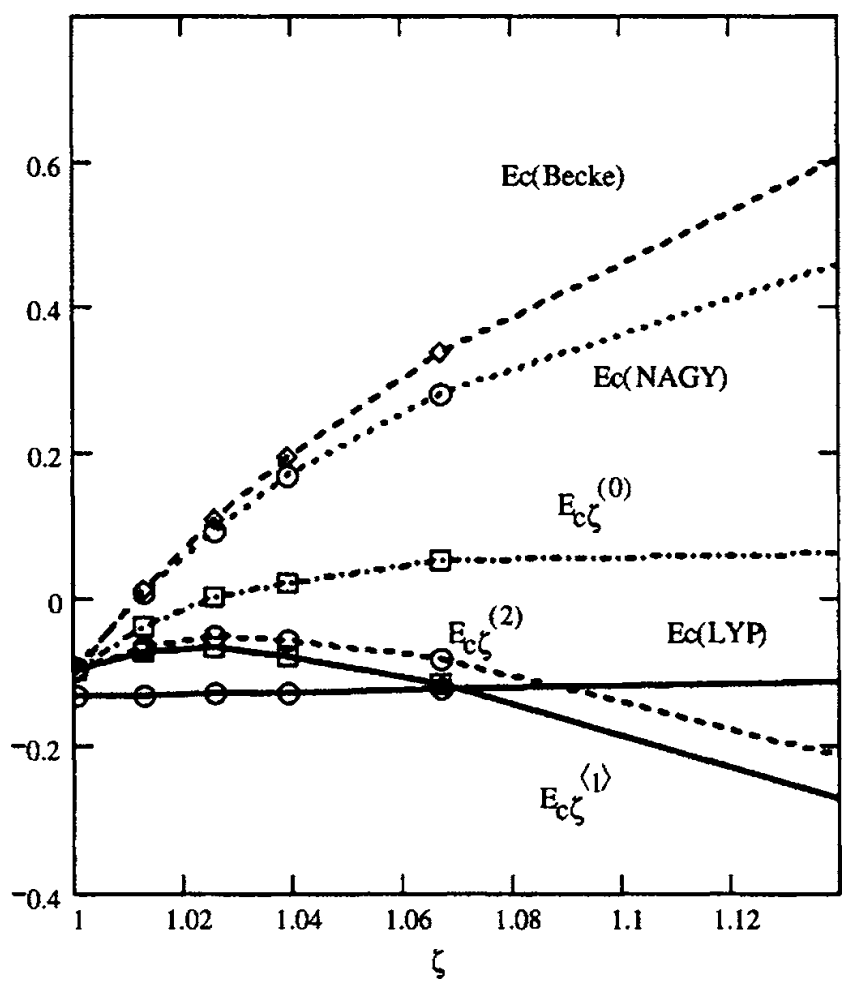

FIG. 6. Plots of $E_{\mathrm{c}}[\rho]$ based on different methods of computing the exchange energy for the $\mathrm{Be}$ atom. Also included for comparison is $E_{\mathrm{c}}^{\mathrm{LYP}}[\rho / \zeta]$, the solid line with circles. The solid line with boxes is the correlation energy $E_{\mathrm{c} \zeta}^{(1)}$. The dot-dashed line with boxes is $E_{\mathrm{c} \zeta}^{(0)}$. The dotted line with circles is $E_{\mathrm{c} \zeta}^{\mathrm{K}} \mathrm{Fg}$ using the 1-matrix to compute the exchange. The dashed line with circles is $E_{\mathrm{c} \zeta}^{(2)}$. The dashed line with diamonds is computed using the Becke exchange formula.

the 1-matrix to compute the exchange energy. Nagy has proposed determining the value of $\zeta$ for which $E_{c}^{\mathrm{Nag}}=0$, then using a more easily determined formula for the exchange energy to determine the total energy. In Table II we list values of $\zeta$ for which $E_{c}^{\mathrm{Nagy}}=0$, as well as values of $E_{\mathrm{c}}$ at $\zeta=1$.

\section{DISCUSSION}

Approximate density functionals which more accurately account for antisymmetry through better exchange potentials tend to produce higher kinetic energies. Density functionals that do not adequately account for the antisymmetry in the wave function tend to have problems with self-interaction. In this article we have isolated the antisymmetry aspect of DFT for the four-electron systems studied by systematically and continuously relaxing the antisymmetry of the Kohn-Sham ensemble through density scaling.

Our work has potential implications for correcting selfinteraction error. An exchange-correlation functional has a self-interaction error arising when there does not exist any antisymmetric wave function with the exchange-correlation energy given by the functional. That is, self-interaction error arises when the exchange-correlation functional is not $N$-representable. ${ }^{4,6,55}$ Clearly for $\zeta>1$, our functionals do not correspond to any fermionic state (because they violate the Pauli principle) and so they are not $N$-representable. The present construction, then, provides an appealingly direct method for measuring the effects of self-interaction error 
and, more importantly, the magnitudes of the corrections that would be needed to "correct" for self-interaction error so that the total energy is appropriately recovered.

Besides the generalized Kohn-Sham scheme having the scaled density, it is possible to define another Kohn-Sham system with the original density and the Pauli principle relaxed. Obviously, one could object to the use of wave functions or density matrices not having the symmetry of the real system. However, see for instance, ${ }^{56-60}$ the symmetry contstraint is often ignored in approximation theories (HartreeFock, DFT, and optimized potential theory).

The kinetic energy and exchange energy are inextricably linked. A high magnitude of the exchange energy results in a high kinetic energy. In the Be isoelectronic series an increase in $\zeta$ relaxes the antisymmetry constraint, resulting in a decrease in both the kinetic energy $T_{\zeta}[\rho]$ and the magnitude of the exchange energy computed either from the ensemble or the $1 s^{2} 2 s^{2}$ determinant. The corresponding diminished exchange hole requires less kinetic energy.

But the exchange energy computed from the $N$-electron 1-matrix in Eq. (29) increases because the occupation number of the $1 s$ orbital goes above 2 . This results in a correlation energy that tends to zero as $\zeta$ is increased slightly above one. This has been shown to be favorable for making approximations for the total energy. ${ }^{15}$

\section{SUMMARY}

We have used density scaling to examine the effects of relaxing the Pauli principle in density functional theory. The Kohn-Sham kinetic energy $T_{s}[\rho]$ is the minimum kinetic energy for the given density $\rho(\mathbf{r})$. Under normal circumstances that kinetic energy is constrained by requiring that it be represented by an antisymmetric wave function. When the antisymmetry constraint is relaxed the kinetic energy decreases as does the magnitude of the exchange energy. Both the magnitude of the exchange energy calculated from the $1 s^{2} 2 s^{2}$ determinant and the kinetic energy decreased as the antisymmetry was relaxed in the four-electron systems studied here. The conjointness of the kinetic energy and the exchange energy results in lower kinetic energies when the magnitude of the exchange energy decreases. As we strive to develop more accurate density functionals it will be necessary to embed more information about antisymmetry and the Pauli principle into those functionals.

\section{ACKNOWLEDGMENTS}

Valuable discussions with professor K. D. Sen are acknowledged. One of the authors (P.W.A.) acknowledges support from the NSERC and the Canada Research Chairs. One of the authors (A.N.) acknowledges support from grant OTKA No. T029469.

\footnotetext{
${ }^{1}$ R. G. Parr and W. Yang, Density Functional Theory of Atoms and Molecules (Oxford University Press, New York, 1989).

${ }^{2}$ Electronic Density Functional Theory: Recent Progress and New Direc-
}

tions, edited by J. F. Dobson, G. Vignale, and M. P. Das (Plenum, New York, 1998).

${ }^{3}$ A. D. Becke, J. Chem. Phys. 122, 064101 (2005)

${ }^{4}$ P. Mori-Sanchez, A. J. Cohen, and W. Yang, J. Chem. Phys. 124, 201102 (2006).

${ }^{5}$ P. Mori-Sanchez, A. J. Cohen, and W. Yang, J. Chem. Phys. 124, 091102 (2006).

${ }^{6}$ P. W. Ayers and S. B. Liu, Phys. Rev. A 75, 022514 (2007).

${ }^{7}$ M. Levy and J. P. Perdew, Phys. Rev. A 32, 2010 (1985).

${ }^{8}$ R. G. Parr and S. Liu, Chem. Phys. Lett. 276, 164 (1997).

${ }^{9}$ S. Liu and R. G. Parr, Chem. Phys. Lett. 278, 341 (1997).

${ }^{10}$ R. G. Parr and S. Liu, Chem. Phys. Lett. 280, 159 (1997).

${ }^{11}$ S. Lui and R. G. Parr, J. Mol. Struct.: THEOCHEM 501, 29 (2000).

${ }^{12}$ S. Liu, F. De Proft, and R. G. Parr, Adv. Quantum Chem. 36, 77 (2000).

${ }^{13}$ G. K.-L. Chan and N. C. Handy, Phys. Rev. A 59, 2670 (1998).

${ }^{14}$ Á. Nagy, Chem. Phys. Lett. 411, 492 (2005).

${ }^{15}$ Á. Nagy, J. Chem. Phys. 123, 044105 (2005).

${ }^{16}$ M. Levy, Proc. Natl. Acad. Sci. U.S.A. 76, 6062 (1979).

${ }^{17}$ E. H. Lieb, Int. J. Quantum Chem. 24, 243 (1983).

${ }^{18}$ M. Levy and J. P. Perdew, NATO ASI Ser., Ser. B 123, 11 (1985).

${ }^{19}$ R. C. Morrison, J. Chem. Phys. 117, 10506 (2002).

${ }^{20}$ P. W. Ayers and W. Yang, J. Chem. Phys. 124, 224108 (2006).

${ }^{21}$ M. Levy, Phys. Rev. A 26, 1200 (1982).

${ }^{22}$ J. P. Perdew, R. G. Parr, M. Levy, and J. L. Balduz, Jr., Phys. Rev. Lett. 49, 1691 (1982).

${ }^{23}$ S. M. Valone, J. Chem. Phys. 73, 4653 (1980).

${ }^{24}$ W. Yang, Y. Zhang, and P. W. Ayers, Phys. Rev. Lett. 84, 5172 (2000).

${ }^{25}$ P. W. Ayers, J. Math. Chem. (in press).

${ }^{26}$ W. Kohn and L. J. Sham, Phys. Rev. 140, A1133 (1965).

${ }^{27}$ Q. Zhao and R. G. Parr, Phys. Rev. A 46, 2337 (1992).

${ }^{28}$ Q. Zhao and R. G. Parr, J. Chem. Phys. 98, 543 (1992).

${ }^{29}$ Q. Zhao, R. C. Morrison, and R. G. Parr, Phys. Rev. A 50, 2138 (1994).

${ }^{30}$ R. G. Parr and L. J. Bartolotti, J. Phys. Chem. 87, 2810 (1983).

${ }^{31}$ P. W. Ayers, Proc. Natl. Acad. Sci. U.S.A. 97, 1959 (2000).

${ }^{32}$ R. C. Morrison and Q. Zhao, Phys. Rev. A 51, 1980 (1995).

${ }^{33}$ E. Fermi and E. Amaldi, Accad. Ital. Rome 6, 117 (1934).

${ }^{34}$ P. W. Ayers, R. C. Morrison, and R. G. Parr, Mol. Phys. 103, 2061 (2005).

${ }^{35}$ R. C. Morrison, J. Chem. Phys. 117, 10506 (2002).

${ }^{36}$ M. Hoffmann-Ostenhof and T. Hoffmann-Ostenhof, Phys. Rev. A 16, 1782 (1977).

${ }^{37}$ N. H. March, Phys. Lett. 113A, 476 (1986).

${ }^{38}$ M. Levy and H. Oui-Yang, Phys. Rev. A 38, 625 (1988).

${ }^{39}$ P. W. Ayers and R. G. Parr, Int. J. Quantum Chem. 95, 877 (2003).

${ }^{40}$ M. Levy, J. P. Perdew, and V. Sahni, Phys. Rev. A 30, 2745 (1984).

41 Á. Nagy, Acta Phys. Hung. 70, 33 (1991) and references therein.

${ }^{42}$ J. A. Flores and J. Keller, Phys. Rev. A 58, 1775 (1998).

${ }^{43}$ J. P. Perdew, M. Ernzerhof, A. Zupan, and K. Burke, J. Chem. Phys. 108, 1522 (1998).

${ }^{44}$ J. C. Slater, Phys. Rev. 81, 385 (1951).

${ }^{45}$ C. Lee, W. Yang, and R. G. Parr, Phys. Rev. B 37, 785 (1988).

${ }^{46}$ A. D. Becke, J. Chem. Phys. 84, 4522 (1986).

${ }^{47}$ A. D. Becke, Phys. Rev. A 38, 3098 (1988).

${ }^{48}$ A. D. Becke, J. Chem. Phys. 98, 1372 (1993).

${ }^{49}$ R. Colle and O. Salvetti, Theor. Chim. Acta 37, 329 (1975).

${ }^{50} \mathrm{R}$. Colle and O. Salvetti, Theor. Chim. Acta 53, 55 (1979).

${ }^{51}$ M. W. Schmidt, K. K. Baldridge, J. A. Boatz et al., J. Comput. Chem. 14, 1347 (1993).

${ }^{52}$ H. Lee, C. Lee, and R. G. Parr, Phys. Rev. A 44, 768 (1991).

${ }^{53}$ N. H. March and R. Santamaria, Int. J. Quantum Chem. 39, 585 (1991).

${ }^{54}$ P. Fuentealba and O. Reyes, Chem. Phys. Lett. 232, 31 (1995).

${ }^{55}$ E. S. Kryachko and E. V. Ludena, Phys. Rev. A 43, 2179 (1991).

${ }^{56}$ S. Thanos and A. K. Theophilou, J. Chem. Phys. 124, 204109 (2006).

${ }^{57}$ Á. Nagy, Phys. Rev. A 66, 22505 (2002).

${ }^{58}$ Á. Nagy, in Fundamentals of Electron Density Matrices and Density Functional Theory in Atoms, Molecules and Solid State, edited by N. I. Gidopoulos and S. Wilson (Kluwer, Dordrecht, 2003), p. 79.

${ }^{59}$ Á. Nagy and C. Amovilli, J. Chem. Phys. 121, 6640 (2004)

${ }^{60}$ Á. Nagy, J. Chem. Phys. 125, $184104(2006)$. 